\title{
- Avaliação da eficiência da prova de evaginação em metacestódeos de Taenia saginata
}

\section{- Evaluation of evagination test efficiency for Taenia Saginata metacestodes}

* Germano Francisco Biondi ${ }^{1}$ - CRMV-SP n ${ }^{0} 1712$

Carlos Henrique Henrique ${ }^{2}$ - CRMV-SP $n^{0} 3248$

Adriano Cesar Oliveira ${ }^{2}$ - CRMV-G0 $\mathrm{n}^{0} 1974$

Alexandra Pastor Castro ${ }^{3}$ - CRMV-SP n 10653

1 Professor Assistente Doutor da Disciplina de Inspeção de Produtos de Origem Animal - FMVZ - UNESP - Botucatu

2 Médicos Veterinários do Laboratório de Produtos Veterinários Ouro Fino Ltda. Ribeirão Preto - SP

3 Médica Veterinária Residente da Disciplina de Inspeção de Produtos de Origem Animal - FMVZ - UNESP - Botucatu
* Faculdade de Medicina Veterinária e Zootecnia UNESP - Botucatu

Departamento de Higiene Veterinária e Saúde Pública

Distrito de Rubião Júnior CEP 18168-000 - Botucatu - SP

\section{RESUMO}

O objetivo deste trabalho consistiu na padronização de uma técnica de evaginação de cisticercos para o esclarecimento de dúvidas relativas ao diagnóstico da cisticercose bovina em estabelecimentos frigoríficos. Segundo normas técnicas estabelecidas pelo RIISPOA (Regulamento de Inspeção Industrial e Sanitária de Produtos de Origem Animal- Brasil, 1980 e 1997), a cisticercose classifica-se como viva, calcificada, localizada ou generalizada. Todavia, não há definição para a forma em degeneração do cisticerco, que na inspeção final se classifica como cisticercose viva para evitar riscos à saúde dos consumidores, já que a inspeção visual por si só não é capaz de identificar o processo degenerativo do cisticerco. Oitenta cisticercos vivos, com características evidentes de viabilidade, foram selecionados para compor o grupo 1 (controle) e 30 cisticercos de aspecto duvidoso constituíram o grupo 2. O grupo 1 foi dividido em oito subgrupos submetidos a diferentes concentrações de bile bovina fresca. Os resultados revelaram que todos os cisticercos evaginaram quando submergidos em meio composto por solução salina a $0,9 \%, 10 \%$ de bile fresca e $2,5 \%$ de RPMI 1640, apresentando $100 \%$ de eficiência (10/10). Os cisticercos do grupo 2 não sofreram evaginação e a degeneração confirmou-se através de ruptura mecânica e exame do conteúdo vesicular.

Palavras chave: Cisticercose, Taenia saginata, Cystucercys bovis, zoonoses.

\section{Introdução}

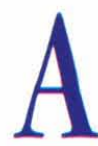

s teníases e cisticercoses representam sério problema sócio-econômico e de saúde pública. No caso específico da $T$. saginata, sua distribuição pode ser considerada mundial, com taxas de pre- valência variáveis nas diferentes áreas geográficas do mundo de acordo com fatores sociais e culturais (ACHA e SZYFRES, 1986).

Em 1947, estimava-se que cerca de 39 milhões de pessoas no mundo já se haviam infectado com a $T$. saginata. Atualmente acredita-se que o número de indi- 
víduos infectados tenha aumentado consideravelmente em virtude do crescimento populacional humano e animal (PAWLOWSKI e SCHULTZ, 1972; ABDUSSALAM, 1975; ACHA e SZYFRES, 1986).

Os casos de cisticercose bovina no Brasil têm apresentado incidência crescente nos últimos anos, conforme se observa em diferentes trabalhos apresentados por CALIL, (1984); REIS et al., (1996) e CARMO et al., (1997). Os referidos estudos basearam-se em levantamento de dados registrados pelo Serviço de Inspeção Federal junto aos estabelecimentos frigoríficos de cada região. Esta zoonose tem sido um fator preocupante para a pecuária de corte em diferentes regiões do país, causando perdas econômicas consideráveis decorrentes das condenações parciais (tratamento pelo frio ou salga) ou totais de carcaças portadoras de cisticercose, acatando as recomendações contidas no RIISPOA (Regulamento de Inspeção Industrial e Sanitária de Produtos de Origem Animal) (BRASIL, 1980, 1997). Ainda devemos considerar os prejuízos econômicos relacionados ao tratamento médico dos portadores humanos e o subdesenvolvimento infantil devido à espoliação parasitária.

É evidente que o problema ocorre pela falta de educação sanitária da população que vive na zona rural e por deficiências de saneamento básico, porém outros fatores de risco contribuem para que o ciclo biológico permaneça em nosso convívio. Dentre eles podemos citar a comercialização e consumo de carne bovina originada de abates clandestinos onde não se realiza nenhum tipo de controle da saúde ou do processamento do animal. Os hábitos culturais revestem-se de grande importância quando determinam o consumo de alimen-

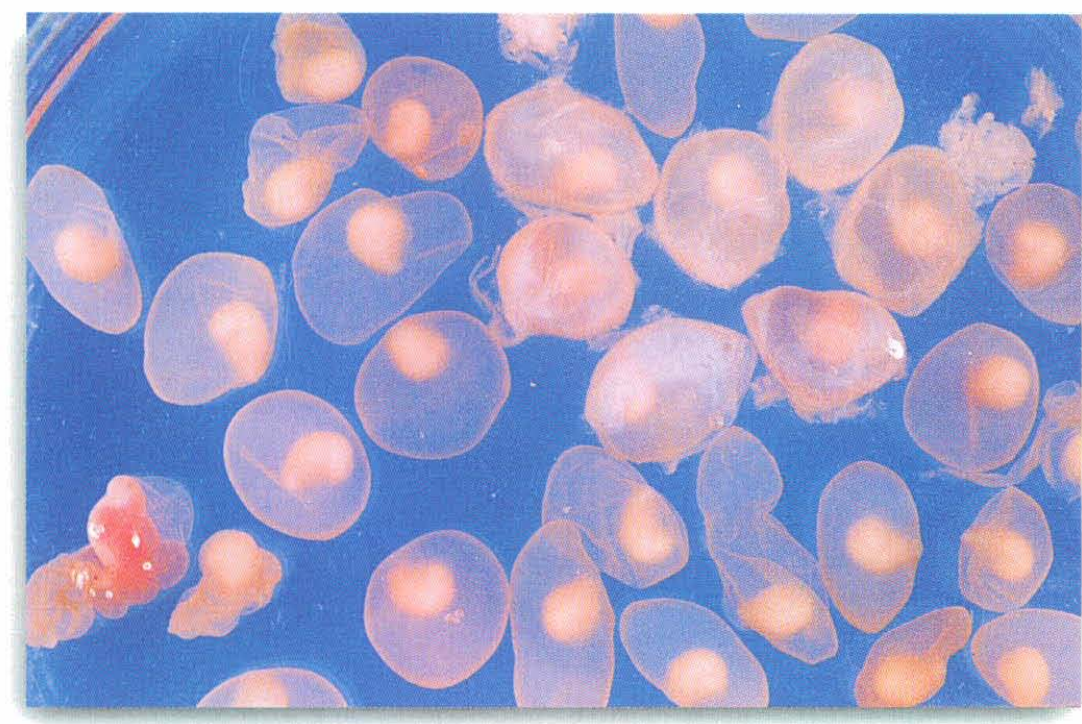

Figura 1: Cysticercus bovis (T. saginata) em diferentes fases de evolução, livres de tecido reacional. tos potencialmente perigosos como a predileção por carne mal passada ou pratos típicos da culinária de cada país como o SHAHLIK da antiga União Soviética e Turquia, o TIKKA da Índia e Paquistão, BASTERMA no Egito, LARB na Tailândia ou o QUIBE cru de origem árabe (MAHAJAN, 1982, SCHENONE et al., 1982, WOODHOUSE et al., 1982, GONZALES LUARCA, 1984; FRISSER, 1988).

Assim, o sistema de vigilância sanitária tem enfatizado ultimamente a importância do médico veterinário e de seus auxiliares na inspeção de bovinos abatidos como guardiões da saúde do homem, detectando carcaças portadoras de cisticercose e determinando a conduta apropriada nesses casos. A atuação do médico veterinário constitui, portanto, uma importante medida preventiva capaz de interromper o ciclo biológico do parasita. Essa função básica dentro do controle do complexo teníasecisticercose é ressaltada por SOUZA et al., (1997).

Durante a inspeção post-mortem de carcaças e vísceras, o médico veterinário e inspetores auxiliares podem deparar-se com diferentes formas de apresentação do cisticerco (Figura 1), que são classificadas em vivas ou calcificadas e localizadas ou generalizadas, de acordo com o aspecto larval e a distribuição na carcaça (BRASIL, 1980, 1997).

Nesses casos, as carcaças e órgãos são encaminhadas ao Departamento de Inspeção Final (DIF) para que se faça uma reavaliação dos achados post-mortem. Quando existem dúvidas quanto ao aspecto do cisticerco (degenerado?), geralmente são classificados como vivos para evitar possíveis riscos à saúde do consumidor, já que a inspeção visual por si só não possibilita identificar o processo degenerativo do cisticerco.

Isso acarretará em perda da lucratividade do frigorífico por gastos adicionais com o tratamento pelo frio dessas carcaças, que poderiam ser destinadas para o consumo imediato.

Frente a essas considerações, o presente trabalho objetivou a padronização de uma técnica rápida e simples para determinar a capacidade de evaginação de metacestódeos de Taenia saginata e, portanto, esclarecer dúvidas relacionadas ao diagnóstico da cisticercose bovina em linhas de abate, sem comprometer a segurança dos consumidores. A técnica de evaginação proposta poderá ainda ser utilizada para o monitoramento da eficiência de drogas antiparasitárias destinadas ao tratamento da cisticercose bovina nos rebanhos de corte. 


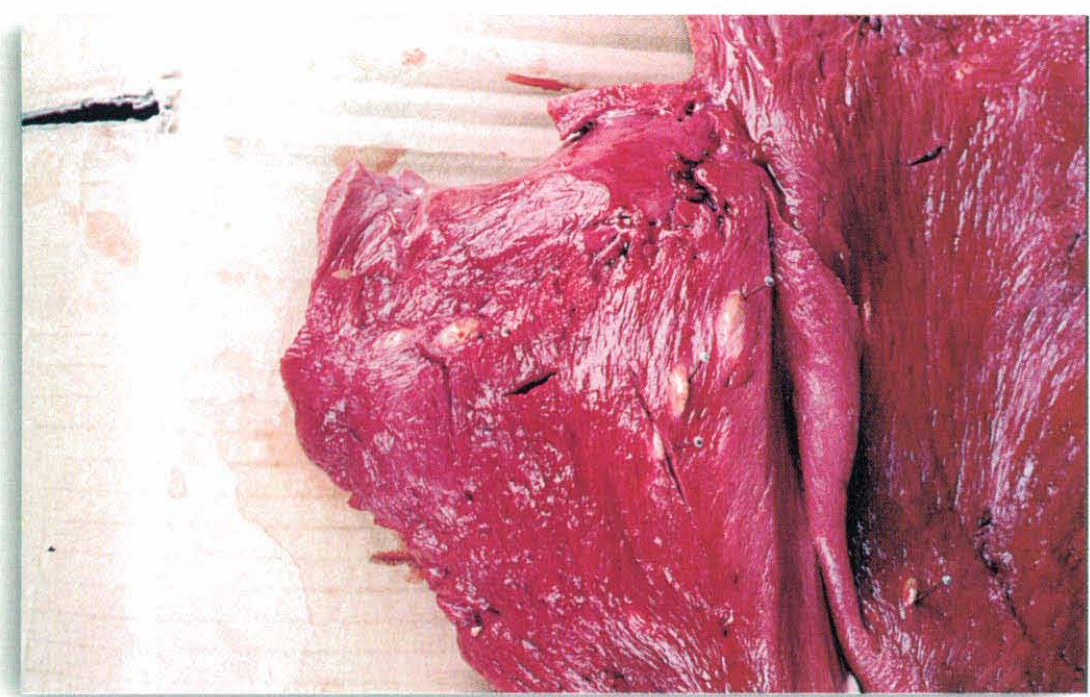

Figura 2: Cysticercus bovis inseridos em músculo cardíaco de bovinos.

\section{Material e Métodos}

\section{Obtenção dos cisticercos}

Os cisticercos utilizados neste experimento (Figura 2) foram obtidos de bovinos abatidos em diferentes frigoríficos do Estado de São Paulo e de Goiás.

Foram colhidos 110 cisticercos confirmados como vivos (viáveis) pelo Serviço de Inspeção Federal durante os exames post-mortem rotineiramente efetuados nos orgãos e respectivas carcaças dos animais (artigo 176, RIISPOA) e reexame dos orgãos (coração e língua) e respectivas carcaças junto ao Departamento de Inspeção Final (DIF). Os fragmentos de tecidos contendo os cisticercos foram colhidos em placa de Petri e, posteriormente, dissecados cuidadosamente, livrando-os totalmente do tecido muscular e cápsula reacional (Figura 1). Selecionaram-se 80 cisticercos de diferentes tamanhos com características evidentes de viabilidade, como aspecto translúcido e límpido, compondo o Grupo 1 . O Grupo 2 (controle) foi constituído pelos 30 cisticercos restantes, considerados de aspecto duvidoso quanto a sua viabilidade, pois notava-se coloração que variava do róseo ao amarelado.

Os cisticercos pertencentes ao Grupo 1 foram subdivididos em 8 subgrupos ( 10 unidades cada) submetidos a testes contendo diferentes concentrações de bile bovina fresca e meio de enriquecimento como descrito no Quadro 1. Todos os cisticercos do Grupo 1 foram individualmente transferidos para tu- bos de ensaio contendo o referido meio e colocados em banho-maria a $37^{\circ} \mathrm{C}$ (Figura 3). Em seguida, com auxílio de lupa, acompanhou-se o processo de evaginação.

Os 30 cisticercos pertencentes ao Grupo 2 foram submetidos ao meio de enriquecimento de maior eficiência, determinado pelo monitoramento do processo de evaginação do Grupo 1.

\section{Meio de enriquecimento}

Empregou-se como componente principal a bile fresca bovina colhida diretamente na sala de matança, acrescida de solução salina $0,9 \%$ e RPMI 1640 (SIGMA) a $2 \%$.

Foram realizadas 8 baterias de testes com diferentes concentrações dos diversos componentes com o intuito de se estabelecer uma padronização das concentrações ideais dos componentes para o processo de evaginação (Quadro 1).

\section{Resultados e Discussão}

O Quadro 2 apresenta os resultados obtidos pelos diferentes testes de evaginação propostos, determinando o número de cisticercos evaginados em cada teste, tempo de evaginação e grau de mobilidade larvar pós evaginação.

A quantificação do grau de mobilidade larvar foi estabelecida por uma escala de pontuação variando de 0 a 5 (sendo $0=$ sem mobilidade e $5=$ mobilidade máxima), baseando-se no início dos movimentos da larva no inte-

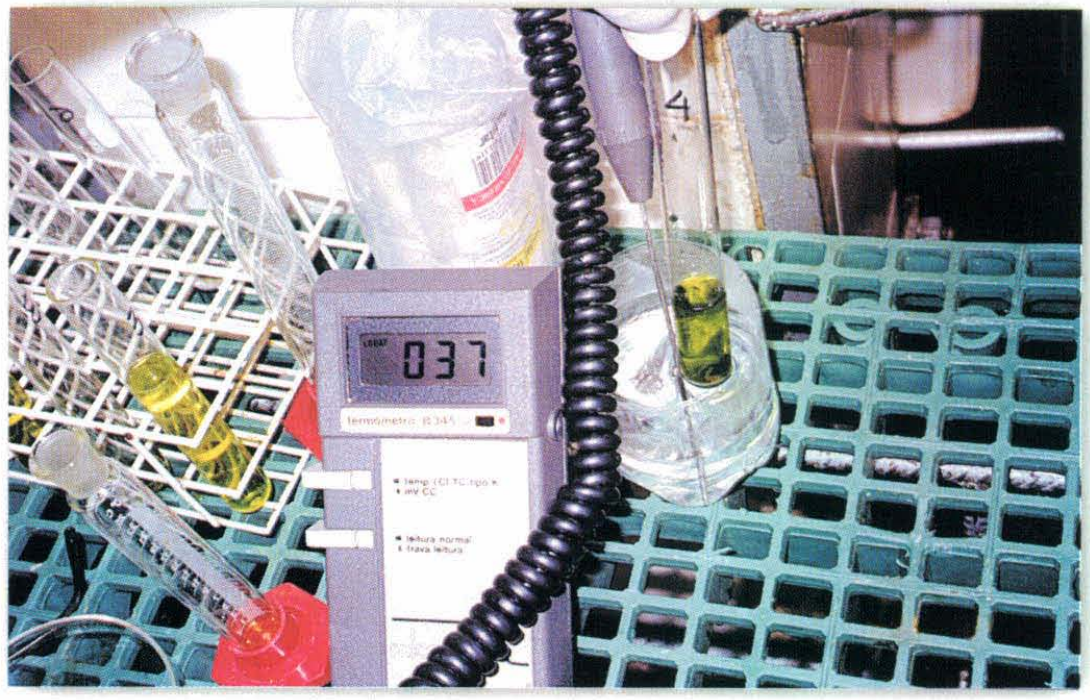

Figura 3: Prova de evaginação - cisticerco imerso em meio de enriquecimento com temperatura monitorada. 
Quadro 1 - Quantificação dos componentes do meio de enriquecimento usado para padronização do teste de evaginação do Cysticercus bovis

\begin{tabular}{|l|c|c|c|c|c|c|c|c|}
\hline COMPONENTES & $\begin{array}{c}\text { TESTE } \\
\mathbf{1}\end{array}$ & $\begin{array}{c}\text { TESTE } \\
\mathbf{2}\end{array}$ & $\begin{array}{c}\text { TESTE } \\
\mathbf{3}\end{array}$ & $\begin{array}{c}\text { TESTE } \\
\mathbf{4}\end{array}$ & $\begin{array}{c}\text { TESTE } \\
\mathbf{5}\end{array}$ & $\begin{array}{c}\text { TESTE } \\
\mathbf{6}\end{array}$ & $\begin{array}{c}\text { TESTE } \\
\mathbf{7}\end{array}$ & $\begin{array}{c}\text { TESTE } \\
\mathbf{8}\end{array}$ \\
\hline Bile bovina fresca & $3 \%$ & $4 \%$ & $5 \%$ & $10 \%$ & $15 \%$ & $20 \%$ & $25 \%$ & $30 \%$ \\
\hline $\begin{array}{c}\text { Solução Salina } \\
\mathbf{0 . 9} \%\end{array}$ & $10 \mathrm{ml}$ & $10 \mathrm{ml}$ & $10 \mathrm{ml}$ & $10 \mathrm{ml}$ & $10 \mathrm{ml}$ & $10 \mathrm{ml}$ & $10 \mathrm{ml}$ & $10 \mathrm{ml}$ \\
\hline RPMI-1640 & $1,0 \%$ & $1,5 \%$ & $2,0 \%$ & $2,5 \%$ & $3,0 \%$ & $3,5 \%$ & $4,0 \%$ & $5,0 \%$ \\
\hline
\end{tabular}

rior da vesícula, tempo de perfuração e intensidade de movimentação pós-evaginação.

Os cisticercos que não evaginaram nos respectivos testes foram rompidos mecanicamente após completarem uma hora de observação. Após rompimento das vesículas, as larvas expostas apresentavam-se com mobilidade.

Os 30 cisticercos pertencentes ao Grupo 2 que foram submetidos ao meio padronizado pelo teste 4 ( $10 \%$ de bile fresca bovina + solução salina 0,9\% + RPMI 1640 a 2,5\%) não evaginaram. Foram rompidos mecanicamente após completarem uma hora e examinados sob lupa. Todos apresentavam-se em diferentes fases de degeneração, com conteúdo fibrosado, de coloração variando do róseo ao amarelo citrino, alguns tendendo à calcificação devido à consistência apresentada.

O processo de desenvolvimento da forma cística do Cysticercus bovis no hospedeiro é bastante variável. Diferentes fatores estão envolvidos (espécie, imunidade, genético, etc.), podendo o cisticerco apresentar-se de diferentes formas no tecido muscular: vesicular límpida com ausência de tecido fibroso, vesicular leitosa com presença de tecido fibroso circunjacente, vesicular rósea com presença de tecido fibroso circunjacente, vesicular amarelado citrino com presença de tecido fibroso, dificultando a diferenciação entre a forma viva e a degenerada pela simples inspeção visual (Figura 2). A quantidade de tecido fibroso presente também varia de intensidade de acordo com o grau da resposta imunitária do hospedeiro.

Diferentes autores têm apresentado estudos inerentes ao processo de evaginação. RICCETI et al., (1974) realizaram estudos em Cysticercus cellulosae ( $T$. solium) e avaliaram percentual, peso, tamanho, e freqüência das evaginações. Em 1998, COSTA et al., (1988) demonstraram experimento referente a testes de evaginação empregando Cysticercus bovis, utilizando meios à base de bile suína comparadas com bile bovina associadas a diferentes meios nutrientes. Obtiveram melhores resultados com a bile homóloga. Trabalhos através de infecção experimental com observação do processo de evaginação têm sido efetuados. (KAMIYA, 1987).

Quadro 2 - Comparativo dos números de cisticercos evaginados, tempo de evaginação, eficiência e grau de mobilidade larvar* pós-evaginação

\begin{tabular}{|l|c|c|c|c|c|c|c|c|}
\cline { 2 - 8 } \multicolumn{1}{c|}{} & $\begin{array}{c}\text { TESTE } \\
\mathbf{1}\end{array}$ & $\begin{array}{c}\text { TESTE } \\
\mathbf{2}\end{array}$ & $\begin{array}{c}\text { TESTE } \\
\mathbf{3}\end{array}$ & $\begin{array}{c}\text { TESTE } \\
\mathbf{4}\end{array}$ & $\begin{array}{c}\text { TESTE } \\
\mathbf{5}\end{array}$ & $\begin{array}{c}\text { TESTE } \\
\mathbf{6}\end{array}$ & $\begin{array}{c}\text { TESTE } \\
\mathbf{7}\end{array}$ & $\begin{array}{c}\text { TESTE } \\
\mathbf{8}\end{array}$ \\
\hline $\begin{array}{l}\text { Tempo de } \\
\begin{array}{l}\text { Evaginação } \\
\text { (minutos) }\end{array}\end{array}$ & - & - & $\mathbf{2 0}$ & 5 & $30^{\prime}-40^{\prime}$ & $30-35^{\prime}$ & $50^{\prime}$ & $45^{\prime}$ \\
\hline Evaginações & 0 & 0 & 2 & 10 & 3 & 2 & 2 & 1 \\
\hline Eficiência & $0,0 \%$ & $0,0 \%$ & $20 \%$ & $100 \%$ & $30 \%$ & $20 \%$ & $20 \%$ & $10 \%$ \\
\hline $\begin{array}{l}\text { *Grau de } \\
\text { mobilidade } \\
\text { (viabilidade }\end{array}$ & 0 & 0 & $2-3$ & $4-5$ & $2-3$ & 3 & 2 & 2 \\
\hline
\end{tabular}


Estudos preliminares dos efeitos de diferentes agentes físicos e químicos sobre a viabilidade do $C$. bovis foram efetuados por GHEBREKIDAN, (1992).

Segundo os resultados apresentados nos Quadros 1 e 2, verifica-se que o teste 4 foi o melhor no que diz respeito à eficiência de evaginação $(100 \%)$, e quanto ao grau de mobilidade (4-5) demonstrando, portanto, que a concentração de $10 \%$ de bile fresca associada ao meio nutriente (RPMI-1640) a 2,5\% é compatível com o teste.

Os estudos apresentados pelos diferentes autores, não fazem referência à aplicabilidade da prova de evaginação como método que possa auxiliar junto aos Serviços de Inspeção, como forma de poder esclarecer situações duvidosas quanto ao grau de viabilidade do cisticer- co, para elucidação de diagnóstico nos casos de cisticercose viva.

\section{Conclusões}

O presente trabalho demonstra que o teste de evaginação poderá, num futuro próximo, servir como ferramenta auxiliar de diagnóstico junto aos órgãos oficiais de Inspeção Industrial e Sanitária, para dirimir dúvidas que muitas vezes são desconsideradas. Além disso, o referido teste poderá ser empregado como forma de monitorar junto aos Serviços de Inspeção, carcaças de animais abatidos, procedentes de regiões endêmicas para cisticercose e que receberam drogas parasiticidas específicas.

\section{SUMMARY}

The objective of this study was to standardize a cysticerci evagination test to be used to clarify doubts related to the diagnosis of cattle cysticercosis in the slaughterhouse. According to the technical rules of the Brazilian Regulation on Industrial and Sanitary Inspection of Animal Products (1980 and 1997), cysticercosis is classified as live, calcified, localized or generalized. However, there is no definition for the degenerated cysticerci. In order to prevent risks to the health of consumers, this presentation is classified as live cysticercosis, as visual inspection alone is not able to identify the degeneration process. Eighty live cysticerci with evident viability characteristics were selected as group 1 (control), divided into eight subgroups, and 30 cysticerci with undefined aspect formed group 2. Group 1 was submitted to different concentrations of fresh cattle bile. In group 1, results showed a $100 \%$ efficiency as all cysticerci (10/10) evaginated when immersed in a medium containing $0.9 \%$ saline, $10 \%$ fresh bile and $2.5 \%$ RPMI 1640. Cysticerci of group 2 did not evaginate and mechanical rupture and examination of vesical contents confirmed their degeneration.

Key words: Cysticercosis, Taenia saginata, Cysticercus bovis, zoonosis.

\section{REFERÊNCIAS BIBLIOGRÁFICAS}

1 - ABDUSSALAM, M. El problema de la teníasis - cisticercosis In: REUNION INTERAMERICANA SOBRE EL CONTROL DE LA FIEBRE AFTOSA Y OTRAS ZOONOSIS, 7. Washington, Organización Panamericana de la Salud, 1975. p.117-29. (Publicación científica, 295)

2 - ACHA, P.N.; SZYFRES, B. Zoonosis y enfermedades transmisibles comunes al hombre $\mathbf{y}$ a los animales. 2 ed. Washington: Organización Panamericana de la Salud, 1986. p.763-74. (Publicaciones científica, 503)

3 - BRASIL Leis, decretos, etc. Ministério da Agricultura. Regulamento de Inspeção Industrial e Sanitária de Produtos de Origem Animal - RIISPOA, aprovado pelo Decreto ${ }^{\circ}$ 30.691, de 29.3.52, alterado pelo Decreto ${ }^{\circ} 1.255$, de 25.6.62. Brasília, 1980. 166p.

4 - BRASIL Leis, decretos, etc. Ministério da Agricultura. Regulamento de Inspeção Industrial e Sanitária de Produtos de Origem Animal - RIISPOA, aprovado pelo Decreto ${ }^{\circ}$ 30.691, de 29.3.52, alterado pelo Decreto ${ }^{\circ} 1.255$, de 25.6.62. Brasília, 1997. 241p.

5 - CALIL, R.M. Situação atual do complexo teníase humana cisticercose no Brasil. Comunicações Científica Fac. Med. Vet. Zootec. Univ. S. Paulo, v.8, p.227-9, 1984. 
6 - CARMO, R.G.; OLIVEIRA, J.V.; BANDINI, O.R.; CARVALHO, J.O.; LIMA, H.W.; REIS,S. Prevalência de cisticercose bovina no Estado de Mato Grosso do Sul. Higiene Alimentar, v.11, p.45-50, 1997.

7 - COSTA, H.M.A.; LIMA, W.S.; GUIMARÃES, M.P. Cysticercus bovis - I. Ensaios de evaginação. Revista Instituto Medicina Tropical, v.30, p.68-71, 1988.

8 - FRISSER A. Neurocysticercosis in Mexico. Parasitology Today, v.4, p.131-6, 1988

9 - GHEBREKIDAN, H. The effect of different chemical and physical agents on the viability of Cysticercus bovis: a preliminary report. Ethiopian Medical Journal., v.30, p.23-31. 1992.

10 - GONZALES-LUARCA, E. Situação atual do complexo teníase humana-cisticercose nas Américas. Comunicações Científicas Fac. Med. Vet. Zootec. Univ. S. Paulo, v.8, p.227-9, 1984

11 - KAMIYA, M.; OOI-HK; OHBAYASHI-M; OW-YANG-CK. Bicephalic larval cestode of Taeniidae from rats in Malaysia. Japanese Journal of Veterinary Research, v.35, p.275-82. 1987.

12 - MAHAJAN, R.C. Geographical distribution of human cysticercosis. In: FLISSER, A. (editor), Cysticercosis: present state of knowledge and perspectives. New York: Academic Pres. 1982. p.39-46.
13 - PAWLOWSKI, Z.S.; SCHULTZ, M.G. Taeniasis and cysticercocis (Taenia saginata) Advances in Parasitology, v.10 p.269-343, 1972.

14 - REIS, D.O.; MUNDIM, M.J.S.; CABRAL, D.D.; COSTACRUZ, J.M. Cisticercose bovina: 15 anos de ocorrência em animais abatidos em Uberlândia, Minas Gerais, Brasil 1977-1993. Higiene Alimentar, v.10, p.33-5, 1996.

15 - RICCETI, R.V.; PANETTA, J.C.; MORENO, A.G. Cisticercose um problema cada vez mais grave. Atualidades Veterinárias, v.3, p.4-9, 1974.

16 - SCHENONE, H. VILLARROEL, F. ROJAS, A., RAMIREZ, R. Epidemiology of human cysticercosis in Latin America. In: FLISSER, A.; WILLMS, K.; LACLETE, J.P.; LARRALDE,C.; RIDAURA, C.; BELTRÁN, F. (editors), Cysticercosis: present state of knowledge and perspectives. New York: Academic Press, 1982, p.25-38.

17 - SOUZA, R.M.; ANTUNES, C.F.; GUATIMOSIM, C.B.; RIBEIRO, R.M.P.; OLIVEIRA, A.L.; SANTOS, W.L.M. A importância do serviço de inspeção federal na vigilância sanitária de alimentos: cisticercose bovina Higiene Alimentar, v.11, 1997.

18 - WOODHOUSE, E.; FLISSER, A.; LARRALDE, C. Seroepidemiology of human cysticercosis in Mexico. In FLISSER A.; WILLMS K.; LACLETE J.P.; LARRALDE C.; RIDAURA C. BELTRÁN, F. (Editors): Cysticercosis: present state of knowledge and perspectives. New York: Academic Press, 1982, p.107-26.

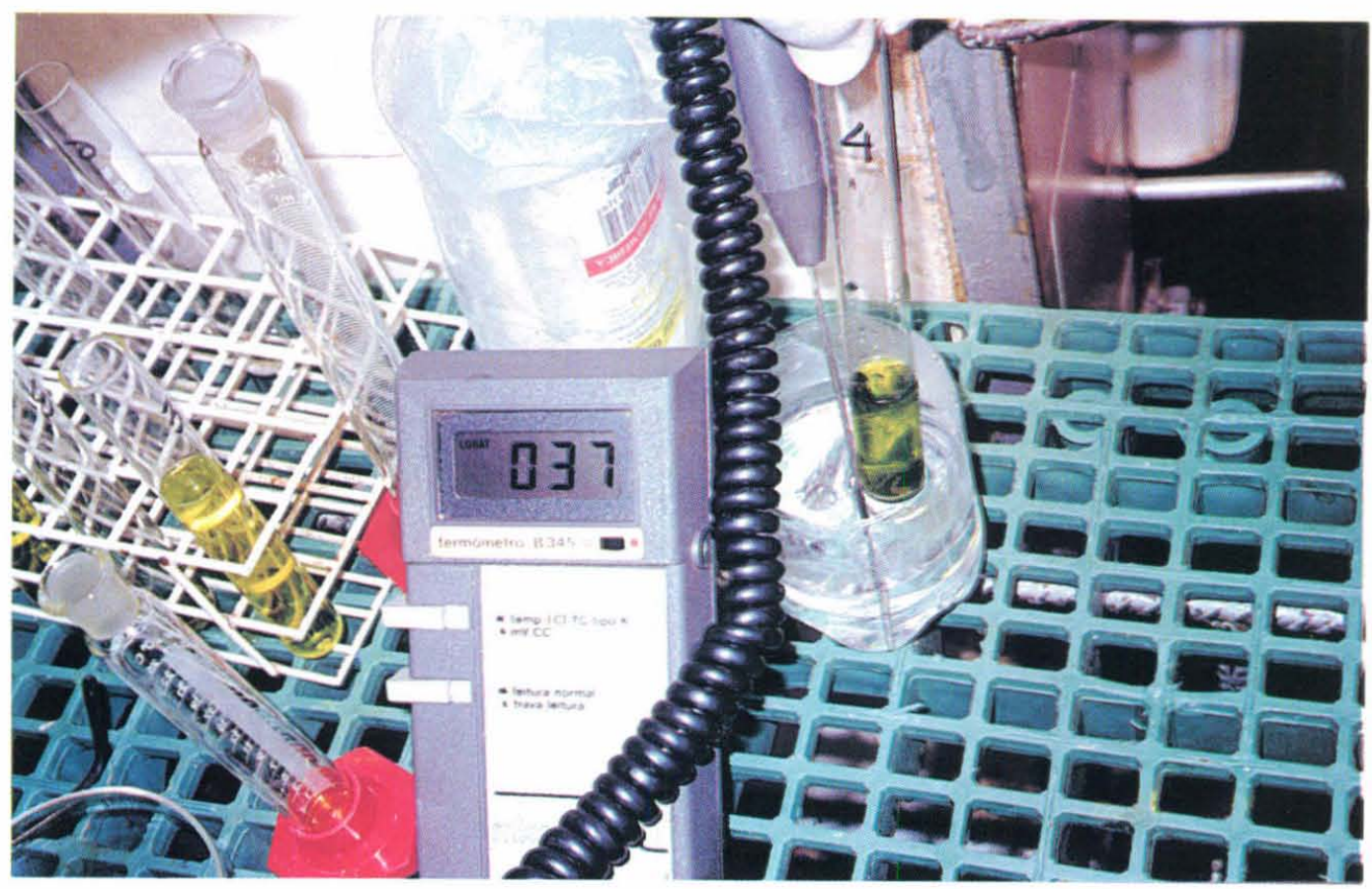

